\title{
Noninvasive Ventilation in Acute Exacerbation of Idiopathic Pulmonary Fibrosis
}

\author{
Toshiki Yokoyama ${ }^{1}$, Yasuhiro Kondoh ${ }^{2}$, Hiroyuki Taniguchi ${ }^{2}$, Kensuke Kataoka ${ }^{2}$, \\ Keisuke Kato ${ }^{2}$, Osamu Nishiyama ${ }^{2}$, Tomoki Kimura ${ }^{2}$, Ryuichi Hasegawa ${ }^{3}$ and Keishi Kubo ${ }^{1}$
}

\begin{abstract}
Background and Objective The outcome of acute exacerbation of idiopathic pulmonary fibrosis (AE-IPF) is usually very poor, and it has been suggested that mechanical ventilation does not benefit AE-IPF patients. Noninvasive ventilation (NIV) has attracted attention as a means to avoid intubation in acute respiratory failure, including acute respiratory distress syndrome (ARDS). This study describes the outcome of patients with AE-IPF who were treated with NIV.

Methods Patients included in the study were those who fulfilled the criteria for AE-IPF during the periods between April 1998 and June 2004 at Tosei General Hospital, and in whom NIV was introduced. Clinical data were obtained retrospectively from patient records.

Results This study included 11 patients. The initial NIV settings were continuous positive airway pressure (CPAP) mode in 6 patients (mean $10.1 \pm 2.5 \mathrm{cmH}_{2} \mathrm{O}$ ) and Spontaneous/Timed mode in 5 (mean inspiratory positive airway pressure/expiratory positive airway pressure; $15.0 \pm 3.3 / 10.2 \pm 2.9 \mathrm{cmH}_{2} \mathrm{O}$ ). Five patients avoided intubation and survived more than 3 months after AE-IPF. Six patients who failed NIV died within 3 months. In these 6 patients, 4 required intubation. The other 2 patients, who refused endotrachial intubation, died without intubation. Median survival time and 3-month survivals after acute exacerbation were 30 days, and $45.5 \%$, respectively.
\end{abstract}

Conclusion Considering extremely poor prognosis of AE-IPF, our findings suggest that NIV is a viable option for the respiratory management in AE-IPF, and should be studied in a large, well-controlled trial.

Key words: ARDS, interstitial lung disease, idiopathic pulmonary fibrosis, ventilation, acute exacerbation of idiopathic pulmonary fibrosis

(Inter Med 49: 1509-1514, 2010)

(DOI: 10.2169/internalmedicine.49.3222)

\section{Introduction}

Idiopathic pulmonary fibrosis (IPF) is the most common form of interstitial lung disease $(1,2)$. Most cases of IPF proceed with relentless but relatively slow deterioration; however, some cases, particularly those of acute exacerbation of IPF (AE-IPF), show a faster progression $(3,4)$. AEIPF has attracted attention because of its survival impact (5), and some investigators have even suggested that mechanical ventilation (MV) does not benefit IPF patients presenting with acute respiratory failure (6).
Recently, noninvasive ventilation (NIV), such as noninvasive positive pressure ventilation (NPPV) and continuous positive airway pressure (CPAP), has been recognized as a means to avoid intubation, reduce mechanical ventilationassociated pneumonia (VAP), and even improve prognosis in selected cases of acute respiratory failure. Antonelli et al. reported that when NIV was applied as first-line intervention in ARDS, intubation could be avoided in $54 \%$ of treated patients in expert centers (7), and avoidance of intubation was associated with less VAP and a lower intensive care unit mortality rate. Hilbert et al. demonstrated that in selected immunosuppressed patients with pneumonitis and acute res-

${ }^{1}$ The First Department of Internal Medicine, Shinshu University School of Medicine, Matsumoto, ${ }^{2}$ Department of Respiratory Medicine and Allergy, Tosei General Hospital, Seto and ${ }^{3}$ Department of Critical Care and Intensive Care Medicine, Tosei General Hospital, Seto Received for publication December 5, 2009; Accepted for publication May 6, 2010 Correspondence to Dr. Hiroyuki Taniguchi, taniguchi@tosei.or.jp 
Table 1. Patient Characteristics on Admission

\begin{tabular}{|c|c|c|c|c|c|}
\hline & & $\begin{array}{l}\text { All cases } \\
\mathrm{n}=11\end{array}$ & $\begin{array}{l}\text { Survivors } \\
\mathrm{n}=5\end{array}$ & $\begin{array}{l}\text { Non-survivors } \\
\mathrm{n}=6\end{array}$ & $\mathrm{p}$-value \\
\hline Age & year & $72.3 \pm 7.7$ & $76.2 \pm 8.3$ & $69.0 \pm 5.8$ & NS \\
\hline Gender & Male/female & $7 / 4$ & $1 / 4$ & $6 / 0$ & NS \\
\hline Body height & $\mathrm{cm}$ & $156.2 \pm 9.2$ & $149.8 \pm 13.4$ & $159.5 \pm 5.1$ & NS \\
\hline Body weight & $\mathrm{kg}$ & $49.2 \pm 15.5$ & $46.8 \pm 18.6$ & $50.8 \pm 14.7$ & NS \\
\hline \multirow[t]{2}{*}{ Smoking history } & yes/no & $8 / 3$ & $2 / 3$ & $6 / 0$ & NS \\
\hline & Pack/year & $50.5 \pm 47.0$ & $26.8 \pm 8.1$ & $58.8 \pm 52.4$ & NS \\
\hline \multicolumn{6}{|c|}{ Treatment before the exacerbation } \\
\hline & Yes/no & $1 / 10$ & $0 / 5$ & $1 / 5$ & NS \\
\hline
\end{tabular}

piratory failure, early initiation of NIV is associated with significant reductions in the rates of intubation and serious complications, and an improved likelihood of survival to hospital discharge (8). Since patients with AE-IPF tend to be treated with immunosuppressive therapy, we speculate that NIV may be effective because it reduces intubation and VAP for IPF-AE.

This study describes the outcome of patients with IPF-AE who were treated with NIV.

\section{Methods}

This study protocol was approved by the Human Ethics Review Committee of Tosei General Hospital. The committee did not require the patients' approval or informed consent for the retrospective review of their records.

\section{General examinations and treatments}

Patients included in the study were those who fulfilled the proposed Japanese Respiratory Society criteria for AEIPF (9) during the period between April 1998 and June 2004 at Tosei General Hospital. IPF was defined according to American Thoracic Society (ATS)/European Respiratory Society (ERS) Consensus Statement Criteria. The criteria for AE-IPF were as follows: During the chronic course of IPF, there was 1) acute worsening of dyspnea within the course of one month, 2) bibasilar honeycombing with newly developing ground glass attenuation and/or consolidation on HRCT scans, 3) deterioration of $\mathrm{PaO}_{2}$ of more than 10 mmHg under the same condition, and 4) exclusion of other known causes of exacerbation, such as pulmonary infection, pneumothorax, malignancy, pulmonary thromboembolism, and heart failure.

Standard microbiological investigations with blood and sputum cultures were performed to exclude pulmonary infection in all patients. Bronchoalveolar lavage (BAL) was performed on admission to rule out infectious disease (10) except in patients with severe pulmonary function impairment before AE, marked honeycombing on HRCT, or rejection of BAL.

High-dose corticosteroid therapy was introduced as general therapy for AE-IPF. Another immunosuppressive ther- apy, such as cycrophosphamide or cycrosporin A, was concurrently or subsequently used.

\section{Ventilatory managements}

NIV was initiated in cases of a respiratory failure of $\mathrm{PaO}_{2} / \mathrm{F}_{\mathrm{I}} \mathrm{O}_{2}$ (P/F) less than 300, unless the patients had contraindications of NIV use such as severe coma and pneumothorax. BiPAP Vision (Respironics Inc, Murrysville, PA, USA) was used for NIV. The initial setting for NIV was CPAP mode and the CPAP level was gradually increased to $12 \mathrm{cmH}_{2} \mathrm{O}$. Pressure support was given if high respiratory frequency or respiratory acidosis was found, and $\mathrm{F}_{\mathrm{I}} \mathrm{O}_{2}$ was set at the lowest value to keep $\mathrm{PaO}_{2}$ at more than $60 \mathrm{mmHg}$.

Endotracheal intubation was performed in patients with any of the following criteria: decreased alertness or major agitation requiring sedation, clinical signs of exhaustion (active contraction of the accessory muscles of respiration with paradoxical abdominal or thoracic motion), hemodynamic instability, cardiac arrest, or refractory hypoxemia.

The criteria for the end of NIV use were defined as follows: 1) $\mathrm{P} / \mathrm{F}>200,2$ ) respiration rate $<20,3)$ clinical improvement of the radiological findings.

\section{Statistical analysis}

Three-month and six-month survival after acute exacerbation were evaluated in this study. Stat View ${ }^{\circledR} 5.0$ (SAS Institute Inc.) was used for analysis in this study. Normally distributed parameters are presented as the standard deviation (SD) of the mean. Differences between survivors and nonsurvivors were compared with the Mann-Whitney U test and Fisher's exact probability test. A P-value less than 0.05 denoted the presence of a significant difference.

\section{Results}

The study included 11 patients. All patients underwent NIV. The clinical characteristics were shown on Table 1. There were 7 men and 4 women, with a mean age of $72.3 \pm$ 7.7 years. There were 3 nonsmokers and 8 smokers with mean pack years of $50.5 \pm 47.0$. Ten patients were diagnosed with IPF before acute exacerbation and one patient was diagnosed with IPF in acute exacerbation. The latter patient 
Table 2. Laboratory Findings on Admission

\begin{tabular}{llllll} 
& & $\begin{array}{l}\text { All cases } \\
\mathrm{n}=11\end{array}$ & $\begin{array}{l}\text { Survivors } \\
\mathrm{n}=5\end{array}$ & $\begin{array}{l}\text { Non-survivors } \\
\mathrm{n}=6\end{array}$ & $\mathrm{p}$-value \\
\hline $\mathrm{WBC}$ & $/ \mathrm{mm}^{3}$ & $10544 \pm 4693$ & $11240 \pm 3410$ & $9675 \pm 6616$ & $\mathrm{NS}$ \\
$\mathrm{Hb}$ & $\mathrm{mg} / \mathrm{dL}$ & $11.7 \pm 2.6$ & $12.8 \pm 2.2$ & $10.4 \pm 2.7$ & $\mathrm{NS}$ \\
$\mathrm{Plt}$ & $\mathrm{x} 10^{4} / \mathrm{mm}^{3}$ & $21.8 \pm 12.1$ & $23.0 \pm 11.8$ & $20.4 \pm 14.2$ & $\mathrm{NS}$ \\
$\mathrm{Alb}$ & $\mathrm{g} / \mathrm{dL}$ & $3.2 \pm 0.6$ & $3.4 \pm 0.6$ & $3.0 \pm 0.6$ & $\mathrm{NS}$ \\
$\mathrm{LDH}$ & $\mathrm{IU} / \mathrm{L}$ & $586.3 \pm 156.9$ & $576.2 \pm 196.3$ & $601.4 \pm 127.6$ & $\mathrm{NS}$ \\
$\mathrm{BUN}$ & $\mathrm{mg} / \mathrm{dL}$ & $13.8 \pm 5.5$ & $14.1 \pm 5.9$ & $13.5 \pm 5.8$ & $\mathrm{NS}$ \\
$\mathrm{Cr}$ & $\mathrm{mg} / \mathrm{dL}$ & $0.6 \pm 0.2$ & $0.7 \pm 0.2$ & $0.6 \pm 0.2$ & $\mathrm{NS}$ \\
$\mathrm{CRP}$ & $\mathrm{mg} / \mathrm{dL}$ & $12.6 \pm 7.9$ & $13.6 \pm 8.5$ & $11.6 \pm 8.1$ & $\mathrm{NS}$ \\
$\mathrm{ANA}$ & $(+/-)$ & $2 / 6$ & $1 / 3$ & $1 / 3$ & $\mathrm{NS}$ \\
$\mathrm{RAHA}$ & $(+/-)$ & $4 / 6$ & $3 / 2$ & $1 / 4$ & $\mathrm{NS}$ \\
$\mathrm{APACHE}$ & $\mathrm{II} \mathrm{score}$ & $14.2 \pm 5.5$ & $14.5 \pm 2.6$ & $16.3 \pm 3.4$ & $\mathrm{NS}$ \\
$\mathrm{PaO}_{2} / \mathrm{F}_{\mathrm{I}} \mathrm{O}_{2}$ & & $138.9 \pm 55.7$ & $156.4 \pm 43.9$ & $129.9 \pm 61.4$ & $\mathrm{NS}$ \\
$\mathrm{PaCO}_{2}$ & & $38.2 \pm 5.3$ & $37.7 \pm 4.3$ & $38.8 \pm 7.1$ & $\mathrm{NS}$ \\
$\mathrm{pH}$ & & $7.427 \pm 0.060$ & $7.434 \pm 0.072$ & $7.418 \pm 0.051$ & $\mathrm{NS}$ \\
\hline
\end{tabular}

*Data are presented as mean \pm SD unless indicated.

Table 3. Respiratory Management for AE-IPF

\begin{tabular}{cllll} 
& $\begin{array}{l}\text { All cases } \\
\mathrm{n}=11\end{array}$ & $\begin{array}{l}\text { Survivors } \\
\mathrm{n}=5\end{array}$ & $\begin{array}{l}\text { Non-survivors } \\
\mathrm{n}=6\end{array}$ & p-value \\
\hline Total duration of NIV (days) & & & & \\
& $5.4 \pm 3.8$ & $8.4 \pm 2.9$ & $2.8 \pm 2.1$ & $\mathrm{NS}$ \\
mode $(\mathrm{CPAP} / \mathrm{ST}$ mode) & & & $3 / 2$ & $\mathrm{NS}$ \\
& $6 / 5$ & $3 / 2$ & $3 / 3$ & $\mathrm{NS}$ \\
$\mathrm{CPAP}\left(\mathrm{cmH}_{2} \mathrm{O}\right)$ & $10.0 \pm 2.5$ & $8.0 \pm 0.0$ & $12.0 \pm 2.0$ & $\mathrm{NS}$ \\
$\mathrm{ST} \quad \operatorname{IPAP}\left(\mathrm{cmH}_{2} \mathrm{O}\right)$ & $15.0 \pm 3.3$ & $14.0 \pm 2.8$ & $15.7 \pm 4.0$ & $\mathrm{NS}$ \\
$\quad$ EPAP $\left(\mathrm{cmH}_{2} \mathrm{O}\right)$ & $10.2 \pm 2.91$ & $9.0 \pm 1.4$ & $11.0 \pm 3.6$ &
\end{tabular}

Duration from introduction of NIV to start of steroid therapy (days)

$2.2 \pm 1.2 \quad 2.2 \pm 1.6$
*Data are presented as mean \pm SD unless indicated.
$\dagger \mathrm{NIV}$, non-invasive ventilation; CPAP, continuous positive airway pressure;
ST, spontaneous and timed mode; IPAP, inspiratory positive airway pressure;
EPAP, expiratory positive airway pressure

had typical HRCT findings for UIP of honeycombing at acute exacerbation. One patient was diagnosed with IPF based on surgical lung biopsy (SLB) and the others without SLB were diagnosed clinically with a definite pattern of usual interstitial pneumonia on high-resolution CT. The mean interval between diagnosis and acute exacerbation was $2.8 \pm 2.4$ years. The baseline laboratory findings of these 11 patients are given in Table 2. There was no significant difference between survivors and non-survivors.

All blood cultures were negative, and neither sputum Gram staining nor culture was contributory. After these diagnostic investigations, broad-spectrum antibiotics were administered as empiric therapy until the offending pathogen was identified or ruled out. BAL was performed during acute exacerbation before antibiotics were introduced in 6 patients. In all 6 cases, BAL fluid studies for routine bacterial organisms, opportunistic pathogens, as well as common viral pathogens revealed no evidence of infection.

The initial setting of NIV was CPAP mode in 6 patients (mean 10.1 $\pm 2.5 \quad \mathrm{cmH}_{2} \mathrm{O}$ ) and Spontaneous/Timed (S/T) mode in 5 patients (mean IPAP/EPAP; 15.0 $\pm 3.3 / 10.2 \pm 2.9$ $\mathrm{cmH}_{2} \mathrm{O}$ ). After diagnosis of AE-IPF, all patients were treated with steroid pulse therapy and/or methyl-prednisolone $2 \mathrm{mg} /$ $\mathrm{kg} / \mathrm{day}$, followed by tapering corticosteroid with or without an immunosuppressant. The time from introduction of NIV to steroid therapy was $2.2 \pm 1.2$ days (Table 3 ).

Duration of NIV was $5.4 \pm 3.8$ days in all cases. Intubation was required in 4 of 11 patients, who failed NIV. And the all 4 patients died during 3 months after AE-IPF. Intubation was avoided in 5 of 11 patients, who survived more than 3 months after AE-IPF. The other 2 patients, who refused endotrachial intubation, died without intubation. All 6 patients who failed NIV died within 3 months because of the progression of respiratory failure (Fig. 1). Median survival time (MST), 3-month survival and 6-month survival after acute exacerbation were 30 days, $45.5 \%$, and $30.0 \%$, respectively (Fig. 2). There were no complications of VAP either in the intubated or in the non-intubated patients. 


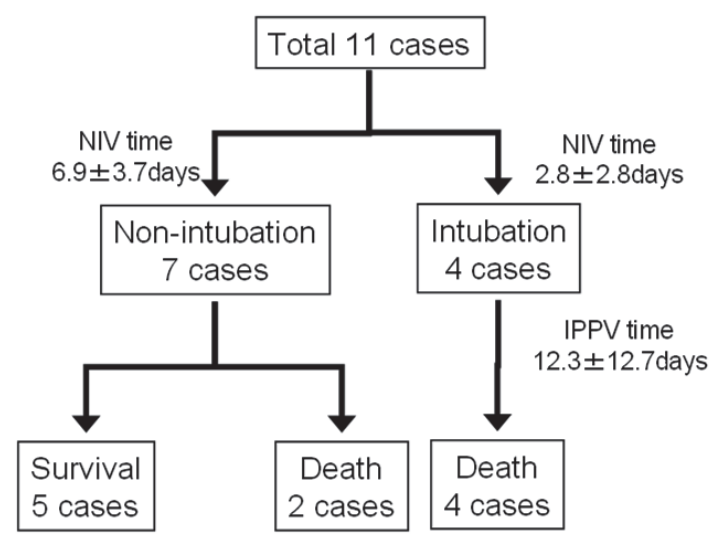

Figure 1. Flow diagram of the process of patients with AEIPF. AE-IPF: acute exacerbation of idiopathic pulmonary fibrosis, NIV: non-invasive ventilation, IPPV: invasive positive pressure ventilation. Data are presented as mean \pm SD unless indicated.

The NIV duration of survivors was significantly longer than that of non-survivors $(8.4 \pm 2.9$ days vs $2.8 \pm 2.1$ days, $\mathrm{p}<$ $0.05)$. There was no significant difference either in the initial $\mathrm{P} / \mathrm{F}$ or in $\mathrm{P} / \mathrm{F}$ change during 1 day management on NIV between survivors and non-survivors (Table 4).

\section{Discussion}

In our case series, we successfully treated about half of the patients with AE-IPF using BiPAP Vision ${ }^{\circledR}$. In our hospital, NIV is used as a first line respiratory management for AE-IPF and high dose corticosteroid therapy is introduced as soon as possible after careful evaluation for possible infectious etiologies. Although a limited number of patients were studied, the results of our strategy may be viewed as rather favorable and acceptable, because the prognosis of AE-IPF has been reported to be extremely poor $(6,11)$, especially in cases requiring mechanical ventilation.

In a report by Stern et al., all the patients but one who successfully received lung transplantation died while receiving mechanical ventilation. They concluded that initiation of MV in IPF patients is of questionable benefit and should, in their opinion, be restricted to patients in whom lungtransplantation can be performed within a few days after initiation of MV (6). Although they did not use the term AEIPF, no infectious etiology was detected in the majority of the patients in their study, and we suppose that AE-IPF might have been included. Bilvet et al. reported similar results among 15 patients who were admitted to an ICU for respiratory failure: 11 patients died in the ICU and 2 died shortly after being discharged from the ICU (11). Considering these findings, the investigators concluded that, except in the rare cases where lung transplantation can be performed shortly after initiation, mechanical ventilation in acute respiratory failure with IPF is of questionable benefit. The hypoxemia in this study was so severe, with a mean

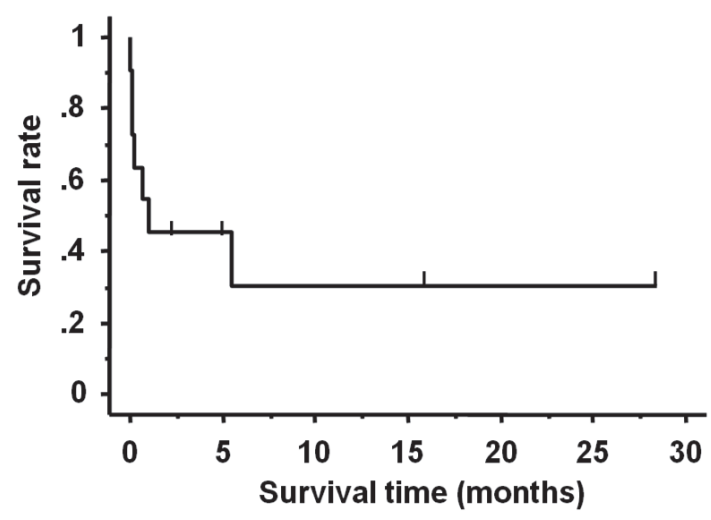

Figure 2. Kaplan-Meier survival curve of patients with acute exacerbation of IPF who received NIV.

$\mathrm{P} / \mathrm{F}$ of 138.9 , that the majority of patients would have required endotracheal intubation if NIV management had not been available. Given the extremely poor prognosis of AEIPF with invasive mechanical ventilation, and our findings that about half of AE-IPF patients could be successfully treated with NIV, NIV seems to be a reasonable option for AE-IPF. Our findings are supported by a recent retrospective study showing that NIV reduced overall in-hospital mortality and ICU stays in a trial of NIV for heterogeneous acute respiratory failure, including interstitial lung disease, in an emergency department (12). In addition, we don't think that we over used NIV even for mild cases, because there was no significant difference of the severity between survivors and non-survivors. Although there was no statistically significant difference in $\mathrm{P} / \mathrm{F}$ ratio on admission between the groups, there was a trend for better $\mathrm{P} / \mathrm{F}$ ratio after 2 hours of NIV among survivors. A larger number of studies may give more conclusive results about the predictive factors for survival at acute exacerbation.

Compared with invasive mechanical ventilation, little is known about the effect of NIV in AE-IPF. In a study by Blivet et al., NIV was used in 5 patients. Two of these patients required intubation and died, one died during NIV, and two had improved conditions and were discharged from the ICU (11). Fumeaux et al. reported that of 11 patients with pulmonary fibrosis who had NIV, all were eventually intubated and died (13). These results differ from ours, but the reason for the difference is not clear. The severity of preexisting IPF and/or of acute respiratory failure, interventions such as high dose steroid therapy, early introduction of interventions, and moderate CPAP/PEEP (10.1/10.2) by NIV are possible reasons.

Mechanical ventilation is an indispensable tool that supports critically ill patients with acute respiratory failure. However, it is also recognized that mechanical ventilation can initiate and exacerbate lung injury, and can contribute to patient morbidity and mortality, a condition recognized as ventilator-induced lung injury (VILI) (14-16). It is reported that patients with IPF may be potentially susceptible to VILI (17). Therefore, prevention of VILI is crucial in the 
Table 4. The $\mathrm{PaO}_{2} / \mathrm{F}_{1} \mathrm{O}_{2}$ Change during 1 Day Management of NIV between Survivors and Non-Survivors

\begin{tabular}{llll} 
& $\begin{array}{l}\text { Survivors } \\
\mathrm{n}=5\end{array}$ & $\begin{array}{l}\text { non-survivors } \\
\mathrm{n}=6\end{array}$ & p-value \\
\hline $\mathrm{PaO}_{2} / \mathrm{F}_{\mathrm{I}} \mathrm{O}_{2}$ & & & \\
Before NIV & $156 \pm 44$ & $129 \pm 61$ & 0.201 \\
2 hours after NIV & $208 \pm 74$ & $153 \pm 70$ & 0.144 \\
24 hours after NIV & $239 \pm 124$ & $192 \pm 83 *$ & 0.882 \\
$*: \mathrm{n}=3$ & & &
\end{tabular}

management of mechanical ventilation. We therefore managed patients with AE-IPF mainly using the CPAP mode with $12 \mathrm{cmH}_{2} \mathrm{O}$ to keep the lung open, and with no pressure support to avoid overinflation if high respiratory frequency or respiratory acidosis was not found. Further studies are needed to evaluate the respiratory strategy of NIV for patients with AE-IPF.

In our study, BiPAP Vision ${ }^{\circledR}$ was used in CPAP mode or $\mathrm{S} / \mathrm{T}$ mode for all patients. One may suppose that CPAP mode is less effective than S/T mode for ARDS. However, because BiPAP Vision ${ }^{\circledR}$ can provide better performance in maintaining a fixed pressure level than NIV by traditional mask CPAP or ICU ventilator (Puritan-Bennett 7200ae and Puritan-Bennett 840; Tyco Healthcare; Mansfield, MA) (18), about half of patients with AE-IPF could be managed successfully by BiPAP Vision ${ }^{\circledR}$ in CPAP mode.

In our study, all four patients who received invasive ventilation as well as NIV died with a mean duration of invasive ventilation of 12.3 days. Therefore, no additional benefit was observed in our series with invasive ventilation. There are two possible explanations for this result: first, invasive ventilation itself provides no additional benefit, and second, delayed intubation leads to poor outcome. Because the prognosis of intubated patients with acute exacerbation of IPF is reported to be extremely poor, the former explanation is more likely.
There are some limitations in this study. First, the study was a retrospective, single center study. The small number of patients and lack of a control group in the study make the results inconclusive. However, the result that almost half of the patients in this study could avoid intubation and survive with the support application of NIV suggests the efficacy of NIV for AE-IPF. In past studies, patients with AEIPF showed poor prognosis of $95.7 \%$ and $86.7 \%$ mortality, even if they had baseline oxygenation (mean P/F: 285 (6) and 113 (11)) similar to that in our study (mean P/F: 138.9). Second, this study included only Japanese patients. Further studies are needed to ascertain whether this result applies equally to other ethnic groups.

In conclusion, our findings suggest that NIV can be a possible option for the management of acute respiratory failure in patients with AE-IPF. Because studies on management of AE-IPF are limited, we think that the present findings on respiratory management for AE-IPF are important. Further prospective and controlled studies with large numbers are needed to assess the efficacy of NIV in AE-IPF.

\section{Acknowledgement}

This study was supported by a grant-in-aid for interstitial lung diseases from the Japanese Ministry of Health, Labor and Welfare.

\section{References}

1. ATS/ERS International multidisciplinary consensus classification of the idiopathic interstitial pneumonias. Am J Respir Crit Care Med 165: 277-304, 2002.

2. Raghu G, Chang J. Idiopathic pulmonary fibrosis: current trends in management. Clin Chest Med 25: 621-636, 2004.

3. Kondoh Y, Taniguchi H, Kawabata Y, et al. Acute exacerbation in idiopathic pulmonary fibrosis. Analysis of clinical and pathologic findings in three cases. Chest 103: 1808-1812, 1993.

4. Martinez FJ, Safrin S, Weycker D, et al; IPF Study Group. The clinical course of patients with idiopathic pulmonary fibrosis. Ann Intern Med 142: 963-967, 2005.

5. Parambil JG, Myers JL, Ryu JH. Histopathologic features and outcome of patients with acute exacerbation of idiopathic pulmonary fibrosis undergoing surgical lung biopsy. Chest 128: 3310-3315, 2005.

6. Stern JB, Mal H, Groussard O, et al. Prognosis of patients with advanced idiopathic pulmonary fibrosis requiring mechanical ventilation for acute respiratory failure. Chest 120: 213-219, 2001.

7. Antonelli M, Conti G, Esquinas A, et al. A multiple-center survey on the use in clinical practice of noninvasive ventilation as a firstline intervention for acute respiratory distress syndrome. Crit Care Med 35: 18-25, 2007.

8. Hilbert G, Gruson D, Vargas F, et al. Noninvasive ventilation in immunosuppressed patients with pulmonary infiltrates, fever, and acute respiratory failure. N Engl J Med 344: 481-487, 2001.

9. Taniguchi H, Ebina M, Kondoh Y, et al. Pirfenidone in idiopathic pulmonary fibrosis. Eur Respir J 2009 Dec 8. [Epub ahead of print].

10. Collard HR, Moore BB, Flaherty KR, et al. Acute exacerbations of idiopathic pulmonary fibrosis. Am J Respir Crit Care Med 176: 636-643, 2007.

11. Blivet S, Philit F, Sab JM, et al. Outcome of patients with idiopathic pulmonary fibrosis admitted to the ICU for respiratory failure. Chest 120: 209-212, 2001.

12. Tomii K, Seo R, Tachikawa $R$, et al. Impact of noninvasive ventilation (NIV) trial for various types of acute respiratory failure in the emergency department; decreased mortality and use of the ICU. Respir Med 103: 67-73, 2009. 
13. Fumeaux $T$, Rothmeier $C$, Jolliet $P$. Outcome of mechanical ventilation for acute respiratory failure in patients with pulmonary fibrosis. Intensive Care Med 27: 1868-1874, 2001.

14. Gajic O, Dara SI, Mendez JL, et al. Ventilator-associated lung injury in patients without acute lung injury at the onset of mechanical ventilation. Crit Care Med 32: 1817-1824, 2004.

15. Amato MB, Barbas CS, Medeiros DM, et al. Effect of a protective-ventilation strategy on mortality in the acute respiratory distress syndrome. N Engl J Med 338: 347-354, 1998.

16. Dreyfuss D, Saumon G. Role of tidal volume, FRC, and end- inspiratory volume in the development of pulmonary edema following mechanical ventilation. Am Rev Respir Dis 148: 11941203, 1993.

17. Fernández-Pérez ER, Yilmaz M, Jenad H, et al. Ventilator settings and outcome of respiratory failure in chronic interstitial lung disease. Chest 133: 1113-1119, 2008.

18. Miyoshi E, Fujino Y, Uchiyama A, et al. Effects of gas leak on triggering function, humidification, and inspiratory oxygen fraction during noninvasive positive airway pressure ventilation. Chest 128: 3691-3698, 2005.

(C) 2010 The Japanese Society of Internal Medicine http://www.naika.or.jp/imindex.html 